\title{
Stabilization of a Class of Bilinear Systems in $\mathbb{R}^{3}$
}

\section{Thouraya $\mathrm{K}^{*}$ and Fehmi M}

Department of Mathematics, University of Sfax, Airport Road Km 0.5 BP 1169.3029 Sfax, Tunisia

\section{Abstract}

In this paper, we investigate the stabilization by means of homogeneous feedback of degree zero of a class of bilinear systems in $\mathbb{R}^{3}$.

Keywords: Stabilization; Bilinear systems; Homogeneous systems; Homogeneous feedback

AMS Classification: 93D15-93D20

\section{Introduction}

Asymptotic stabilization of nonlinear control systems has been a subject of researches for several years [1]. The main motivating factor is to better the inadequate existing theories of control system analysis in order to solve some problems concerning robotics, advanced aircraft, smart structures [2]. The stabilization problem of bilinear systems is one of the most important methods to study and understand the stabilization of nonlinear systems. In general, these bilinear systems have the following structure:

$$
\left\{\begin{array}{l}
\dot{x}=A x+u B x . \\
A, B \in M_{n}(\mathbb{R}), x \in \mathbb{R}^{n} \text { and } u \in \mathbb{R}
\end{array}\right.
$$

In addition to the rich mathematical structure that motivates the problem, we can consider this as a stabilization problem for systems with a first order singularity at an equilibrium point $[3,4]$. Vector fields $A x$ and $B x$ are the first order approximations of the state and the input vector fields. We will illustrate this aspect by considering a numerical example. More precisely, we investigate the stabilization of a class of a bilinear system in eqn. (1) in three dimension. We suppose that the surface containing invariant straight lines is a sub manifolds with special algebraic equation $[5,6]$.

In this subject, there has been lot of works about homogeneous systems and for a 'philosophical' introduction to this research field [7-9].

Many searches are edited in the mentioned subject, we can cite Baciotti and Boieri [1] who gives a complete classification of such systems in the plane, Jerbi and Kharrat [5] who give a complete classification of polynomial homogeneous systems in the plane. Jerbi et al. [4] consider a class of bilinear systems in dimension three which can be an extension of another one in R2. They prove that there exists some homogeneous feedback of degree zero stabilizing the considered class if and only if these feedbacks are constants. Luesink and Nijmeijer [9] who give a sufficient condition for constant feedback stabilization (by continuous feedback control) of a class of bilinear systems.

The authors give an algebraic classification of the stabilization of the homogeneous two dimensional systems [5].

$$
\dot{x}=P(x)+u Q(x)
$$

where $\mathcal{P}$ and $\mathcal{Q}$ are homogeneous polynomials having no common linear linear factor. They give many tools to compute explicitly the stabilizing feedback. In [7], the authors study the stabilization of the system 2, in the case P and Q have common linear factor. The same results are generalized in three dimensions [8].
In the present work, we consider the problem of explicitly constructing of a feedback laws $u(x)$ which is homogeneous of degree zero and asymptotically stabilizes the system in eqn. (1). The fundamental idea is that if a vector field $X$ of the closed loop system by a homogeneous feedback (defined on $\mathbb{R}^{3}$ )

$$
\dot{x}=A(x)+u(x) B x
$$

is homogeneous, then it induces a dynamical system on a lower dimensional space: the unit sphere $\mathcal{S}^{2}$. The main tool of this paper will be the theorem of Coleman [2] which gives necessary and sufficient conditions for global asymptotic stability (G.A.S.) of a homogeneous system in 3 -space.

\section{Preliminaries}

In the next, let us consider the system

$$
\dot{x}=X(x)
$$

where $x \in \mathbb{R}^{3}$ and $X$ is a homogeneous vector field (not necessarily polynomial) of odd degree.

In the following, $\|$.$\| and \langle$,$\rangle will denote respectively for the usual$ two-norm and the standard inner product on $\mathbb{R}^{n}$,

$\left(\right.$ for $\mathrm{x} \in \mathbb{R}^{\mathrm{n}}\left(\|x\|=\sqrt{x_{1}^{2}+\ldots+x_{n}^{2}},\langle x, y\rangle=\sum_{i=1}^{n} x_{i} y_{i}\right)$.

Let $x \in \mathbb{R}^{3} \backslash\{0\}, \gamma=\|x\|$ and $y=\frac{x}{r}$. Differentiating $x=r y$ and denoting $r^{m-1}(t) d t=d \tau$, we obtain a system on the unit sphere $\mathcal{S}^{2}$ by writing the equation satisfied by $\dot{y}$.

$$
\dot{y}=X(y)-\langle X(y), y\rangle y
$$

Coleman showed the following theorem.

\section{Theorem 1 (Coleman)}

The origin is an asymptotically stable equilibrium point for the system in eqn.(4) if and only if the following conditions are satisfied.

${ }^{*}$ Corresponding author: Thouraya K, Department of Mathematics, University of Sfax, Airport Road Km 0.5 BP 1169.3029 Sfax, Tunisia, Tel: +216 74244 423; E-mail: thouraya.kharrat@fss.rnu.tn

Received November 05, 2018; Accepted January 11, 2019; Published January 19, 2019

Citation: Thouraya K, Fehmi M (2019) Stabilization of a Class of Bilinear Systems in $\mathbb{R}^{3}$. J Appl Computat Math 8: 433. doi: 10.4172/2168-9679.1000433

Copyright: (c) 2019 Thouraya K, et al. This is an open-access article distributed under the terms of the Creative Commons Attribution License, which permits unrestricted use, distribution, and reproduction in any medium, provided the original author and source are credited. 
1. $\langle X(y), y\rangle<0$ for all the equilibrium points of in eqn.(5).

2. $\int_{0}^{T}\langle X(y(s)), y(s)\rangle d t<0$ for any periodic solution $y(s)$ of the system in eqn.(5); ( $T$ denotes the period of $y(s))$.

\section{Definition 1}

Let $\mathcal{N}$ a subset of $\mathbb{R}^{3}$, we say that $\mathcal{N}$ is an invariant set by the trajectories of system (4) if:

For all $y_{0} \in \mathcal{N}$ one has $c_{\mathrm{t}}\left(y_{0}\right) \in \mathcal{N}$ for all $t \in \mathbb{R}\left(c_{t}\left(y_{0}\right)\right.$ is the solution of equation $\dot{x}=X(x)$ and $\left.c_{0}\left(y_{0}\right)=y_{0}\right)$.

\section{Remark 1}

If $\mathcal{N}$ is a sub manifold of $\mathbb{R}^{3}$ one has: $\mathcal{N}$ is an invariant set by the trajectories of system (4) if:

For all $\mathrm{y}_{0} \in \mathcal{N}$ one has $\mathrm{X}(\mathrm{y}) \in T_{y} \mathcal{N}\left(T_{\mathrm{y}} \mathcal{N}\right.$ is the tangent space of $\mathcal{N}$ at the point $y$ ).

\section{Main Results}

We consider the bilinear system

$\left\{\begin{array}{l}\dot{x}=A x+u B x \\ A, B \in \mathcal{M}_{3}(\mathbb{R}), x \in \mathbb{R}^{3} \text { and } u \in \mathbb{R}\end{array}\right.$

In any basis of $\mathcal{R}^{3}$, matrices $A$ and $B$ take the following forms

$$
A=\left(\begin{array}{lll}
a_{11} & a_{12} & a_{13} \\
a_{21} & a_{22} & a_{23} \\
a_{31} & a_{32} & a_{33}
\end{array}\right) \text { and } B=\left(\begin{array}{lll}
b_{11} & b_{12} & b_{13} \\
b_{21} & b_{22} & b_{23} \\
b_{31} & b_{32} & b_{33}
\end{array}\right)
$$

The closed loop system in eqn.(6) by a homogeneous feedback of degree zero $v(x)$ is

$$
\dot{x}=A x+v(x) B x=X(x)
$$

The vector field $X$ can be written as

$$
X(x)=\left(\begin{array}{l}
X_{1}(x) \\
X_{2}(x) \\
X_{3}(x)
\end{array}\right)=\left(\begin{array}{l}
A_{1}(x)+v(x) B_{1}(x) \\
A_{2}(x)+v(x) B_{2}(x) \\
A_{3}(x)+v(x) B_{3}(x)
\end{array}\right)
$$

with $\mathrm{A}_{i}(x)=\mathrm{a}_{i 1} x_{1}+a_{\mathrm{i} 2} x_{2}+a_{\mathrm{i} 3} x_{3}$ and $B_{i}(x)=b_{i 1} x_{1}+b_{i 2} x_{2}+b_{i 3} x_{3}$, for $i=1 ; 2 ; 3$. Since $X$ is homogeneous of degree one, we can associate a vector field $Y$ defined in the unit sphere $\mathbf{S}^{2}$ by

$Y(y)=X(y)-\langle X(y), y\rangle y$

If $y_{0}$ is an equilibrium point of $Y$, then $Y\left(y_{0}\right)=0$, so $X\left(y_{0}\right)=\left\langle\mathrm{X}\left(y_{0}\right)=\left(y_{0}\right), y_{0}\right\rangle$ The following lemma gives a localization of the equilibrium points of the system

$$
\dot{y}=Y(\mathrm{y})
$$

\section{Lemma 1}

The equilibrium points of $Y$ are contained in the surface

$$
\mathcal{M}=\left\{x \in \mathbb{R}^{3}: \operatorname{det}(A x, B x, x)=0\right\}
$$

Proof: It is clear that if $y$ is an equilibrium point of $Y$, then $Y(y)=0$. So

$$
\mathrm{X}(\mathrm{y})=\langle\mathrm{X}(\mathrm{y}), \mathrm{y}\rangle \mathrm{y}
$$

Taking into account the form of $X$, we get

$$
\text { Ay }+v(y) B y=h X(y) ; \text { yiy }
$$

Finally the vectors $\{A y, B y, y\}$ are linearly dependant and det $(A y, B y, y)=0$.

In the next, we introduce the following homogeneous functions:

$$
\begin{aligned}
& \mathcal{G}(x):=\operatorname{det}\left(\begin{array}{ll}
B_{1 \mathcal{F F}}(x) & x_{1} \\
B_{2}(x) & x_{2}
\end{array}\right), \\
& \mathcal{H}(x):=\operatorname{det}\left(\begin{array}{ll}
A_{1}(x) & x_{1} \\
A_{2}(x) & x_{2}
\end{array}\right), \\
& \phi(x)=\operatorname{det}\left(\begin{array}{ll}
X_{1}(x) & x_{1} \\
X_{2}(x) & x_{2}
\end{array}\right)=\mathcal{H}(x)+v(x) \mathcal{G}(x),
\end{aligned}
$$

and

$$
\mathcal{F}(x):=\operatorname{det}\left(\begin{array}{cc}
A_{1}(x) & B_{1}(x) \\
A_{2}(x) & B_{2}(x)
\end{array}\right)
$$

These functions $\mathcal{G}, \mathcal{H}, \phi$ and $\mathcal{F}$ have an important role in determining the invariant lines of system in eqn.(6), thus the equilibrium points of system in eqn. (7) and the construction of feedback $v$.

so, we shoos $\mathcal{F}(\mathrm{x}) \neq 0$ for all $x \in \mathbb{R}^{3} \backslash\{0\}$, if $\mathcal{F}(\mathrm{x})=0$ we look at $\tilde{\mathcal{F}}(x):=\operatorname{det}\left(\begin{array}{ll}A_{1}(x) & B_{1}(x) \\ A_{3}(x) & B_{3}(x)\end{array}\right)$.

If $\tilde{\mathcal{F}}(x)=0$ we have $A_{1}(x)=\lambda B_{1}(x) A_{2}(x)=\lambda B_{2}(x)$ and $A_{3}(x)=\lambda B_{1}(x)$ the system in eqn. (6) becomes $\dot{x}=(\lambda+u) B x$. this system can be stable with a constant feedback.

\section{Lemma 2}

Let $x \in \mathbb{R}^{3} \backslash\{0\}$ a point such that $\mathcal{F}(x) \neq 0$ and $\left(x_{1}, x_{2}\right) \neq(0,0)$.

$y=\frac{x}{\|x\|}$ is an equilibrium point of $Y$ if and only if

$y \in \mathcal{M} \cap S^{2}$ and $\varphi(x)=0$.

Proof: If $y$ is an equilibrium point of $Y$, then by Lemma 1 $X(y)=v y, v \in \mathbb{R},(<y>$ is an invariant straight line by the system $\dot{x}=X(\mathrm{x})=\mathrm{A}(x)+v(x) B x)$. From Lemma 5.1 the point $y$ is in the set $\mathcal{M} \cap \mathbf{S}^{2}$ and we have

$$
\Phi(x)=\operatorname{det}\left(\begin{array}{ll}
X_{1}(x) & x_{1} \\
X_{2}(x) & x_{2}
\end{array}\right)=0
$$

Inversely if $x$ verifies

$\mathrm{y} \in \mathcal{M} \cap S^{2}$ and $\varphi(x)=0$

Then there exists $\left(\alpha_{1}, \alpha_{2}\right) \neq(0,0)$ such that $\alpha_{1}\left(\begin{array}{l}X_{1}(x) \\ X_{2}(x)\end{array}\right)+\alpha_{2}\left(\begin{array}{l}x_{1} \\ x_{2}\end{array}\right)=\left(\begin{array}{l}0 \\ 0\end{array}\right)$. From the assumption such that $\left(x_{1}, x_{2}\right) \neq(0,0)$, we can deduce that $\alpha_{1} \neq 0$. The point $x$ satisfies the condition $\mathcal{F}(x):=\operatorname{det}\left(\begin{array}{ll}A_{1}(x) & B_{1}(x) \\ A_{2}(x) & B_{2}(x)\end{array}\right) \neq 0$, then the family $\left\{\left(\begin{array}{l}A_{1}(x) \\ A_{2}(x)\end{array}\right),\left(\begin{array}{l}B_{1}(x) \\ B_{2}(x)\end{array}\right)\right\}$ is a basis of $\mathbb{R}^{2}$, If $\mathrm{a}_{2}=0$ we obtain

$$
\alpha_{1}\left(\begin{array}{l}
X_{1}(x) \\
X_{2}(x)
\end{array}\right)=\alpha_{1}\left(\begin{array}{l}
A_{1}(x) \\
A_{2}(x)
\end{array}\right)+\alpha_{1} v(x)\left(\begin{array}{l}
B_{1}(x) \\
B_{2}(x)
\end{array}\right)=\left(\begin{array}{l}
0 \\
0
\end{array}\right)
$$

Which is absurd, then $\alpha_{2} \neq 0$ 
Using the fact $x \in \mathcal{M}$, we can deduce that there exists $\left(\beta_{1}, \beta_{2}, \beta_{3}\right) \neq(0,0,0)$ such that $\beta_{1} A x+\beta_{2} B x+\beta_{3} x=0$. It is clear that

$\beta_{1}\left(\begin{array}{l}A_{1}(x) \\ A_{2}(x)\end{array}\right)+\beta_{2}\left(\begin{array}{l}B_{1}(x) \\ B_{2}(x)\end{array}\right)+\beta_{3}\left(\begin{array}{l}x_{1} \\ x_{2}\end{array}\right)=\left(\begin{array}{l}0 \\ 0\end{array}\right)$

Using the argument that $\left\{\left(\begin{array}{l}A_{1}(x) \\ A_{2}(x)\end{array}\right),\left(\begin{array}{l}B_{1}(x) \\ B_{2}(x)\end{array}\right)\right.$ is a basis of $\mathbb{R}^{2}$ and $\left(x_{1}, x_{2}\right) \neq(0,0)$, we can deduce that $\beta_{3} \neq 0$, we have

$$
\alpha_{1}\left(\begin{array}{l}
A_{1}(x) \\
A_{2}(x)
\end{array}\right)+\alpha_{1} v(x)\left(\begin{array}{l}
B_{1}(x) \\
B_{2}(x)
\end{array}\right)+\alpha_{2}\left(\begin{array}{l}
x_{1} \\
x_{2}
\end{array}\right)=\left(\begin{array}{l}
0 \\
0
\end{array}\right)
$$

We deduce that

$\frac{\beta_{1}}{\beta_{3}}=\frac{\alpha_{1}}{\alpha_{2}}$ and $\frac{\beta_{2}}{\beta_{3}}=v(x) \frac{\alpha_{1}}{\alpha_{2}}$

and

$A x+v(x) B x+\frac{\alpha_{2}}{\alpha_{1}}=0$

Finally one has $Y(y)=-\frac{\alpha_{2}}{\alpha_{1}} y$ and $\mathrm{y}$ is an equilibrium point of $\mathrm{Y}$.

Proposition 1: Suppose that the equilibrium points of $Y$ satisfy $F(y) \neq 0$. The vector field $Y$ satisfies to the first condition of Coleman theorem if and only if

$\Phi(y)=0$ and $\frac{\mathcal{F}(y)}{\mathcal{G}(y)}>0$

Proof: Let $x \in \mathbb{R}^{3} \backslash\{0\}$ and $y=\frac{x}{\|x\|}$. Notice that if $y$ is an equilibrium point of $Y$ then $\mathrm{X}(x)=v x .(v=\langle\mathrm{X}(x), x\rangle)$ and

$$
\left(\begin{array}{l}
A_{1}(x) \\
A_{2}(x)
\end{array}\right)+v(x)\left(\begin{array}{l}
B_{1}(x) \\
B_{2}(x)
\end{array}\right)=v\left(\begin{array}{l}
x_{1} \\
x_{2}
\end{array}\right)
$$

Therefore

$$
\left(\begin{array}{ll}
A_{1}(x) & B_{1}(x) \\
A_{2}(x) & B_{2}(x)
\end{array}\right)\left(\begin{array}{l}
1 \\
v(x)
\end{array}\right)=v\left(\begin{array}{l}
x_{1} \\
x_{2}
\end{array}\right)
$$

and the fact $F(y) \neq 0$, one has

$$
\begin{aligned}
& \left(\begin{array}{l}
1 \\
v(x)
\end{array}\right)=v\left(\begin{array}{ll}
A_{1}(x) & B_{1}(x) \\
A_{2}(x) & B_{2}(x)
\end{array}\right)^{-1}\left(\begin{array}{l}
x_{1} \\
x_{2}
\end{array}\right) \\
& \text { Thus } 1=v \frac{-\mathcal{F}(x)}{\mathcal{G}(x)} \cdot \text { Finally } \\
& v=<X(x), x>=\frac{-\mathcal{G}(x)}{\mathcal{F}(x)}
\end{aligned}
$$

The vector field $Y$ satisfies the first condition of Coleman theorem if $y$ is an equilibrium point of $Y$ then $v=\langle X(x), x\rangle<0$. All this is equivalent to the writing from Lemma 5.2 that if $\Phi(x)=0$ then $<X(x), x><0$ which is equivalent to the following

$$
\frac{\mathcal{F}(x)}{\mathcal{G}(x)}>0
$$

\section{Theorem 2}

If the vector field $X$ has an invariant linear plane $\mathcal{P}$ and all the straight lines of $X$ are contained in $\mathcal{P}$, then the system in eqn.(7) don't have any periodic orbit.

Proof: It is clear that all equilibrium points of the vector field $Y$ lie in the circle $\mathcal{C}=\mathcal{P} \cap \mathcal{S}^{2}$. Since the plane $\mathcal{P}$ is invariant by the flows of the vector field $X$, then the circle $\mathcal{C}$ is invariant by the flows of the vector field $Y$. Any periodic orbit of the vector field $Y$ can be located in one of the two regions delimited by circle C. Using the theorem of Poincar'eBendixson, that in the interior of any periodic orbit on $\mathbf{S}^{2}$ there exists an equilibrium point. However, we have seen that the only equilibrium points are located on the circle $\mathcal{C}$, then there are no periodic orbits.

\section{Construction of the Function Feedback $v$}

We define the following homogeneous polynomial function of degree 3

$$
\mathcal{D}\left(x_{1}, x_{2}, x_{3}\right)=\operatorname{det}\left(\begin{array}{lll}
A_{1}(x) & B_{1}(x) & x_{1} \\
A_{2}(x) & B_{2}(x) & x_{2} \\
A_{3}(x) & B_{3}(x) & x_{3}
\end{array}\right)
$$

Since $\mathcal{D}$ is a homogeneous polynomial of degree 3,we suppose that:

$\mathcal{D}\left(x_{1}, x_{2}, x_{3}\right)=\left(x_{3}-a_{1} x_{1}-b_{1} x_{2}\right) \mathrm{R}\left(x_{1}, x_{2}, x_{3}\right)$

Or

$$
\mathcal{D}\left(x_{1}, x_{2}, x_{3}\right)=\left(x_{3}-a_{1} x_{1}-b_{1} x_{2}\right)\left(x_{3}-a_{2} x_{1}-b_{2} x_{2}\left(x_{3}-a_{3} x_{1}-b_{3} x_{2}\right)\right.
$$

such that $R$ is a definite homogeneous function of degree 2 .

The construction of the feedback $\mathrm{v}$ depends on the geometric structure of the set $M$ defined by $\mathcal{M}=\left\{\mathrm{x} \in \mathbb{R}^{3}: \mathcal{D}\left(x_{1}, x_{2}, x_{3}\right)=0\right\}$, that contains the invariant straight lines by the system in eqn.(6).

We suppose that $\mathcal{P}_{i}: x_{3}=a_{i} x_{1}+b_{i} x_{2} ; a_{1} \in \mathbb{R}, b_{1} \in \mathbb{R}$ for $i=1,2,3$ is an invariant linear plane of the vector fields $A$ and $B$. The restriction of $A+v B$ on the plane $\mathcal{P}_{i}$ is following system

$$
\left\{\begin{array}{l}
\dot{x}_{1}=A_{1}\left(x_{1}, x_{2}, a_{1} x_{1}+b_{1} x_{2}\right)+v B_{1}\left(x_{1}, x_{2}, a_{i} x_{1}+b_{i} x_{2}\right) \\
\dot{x}_{2}=A_{2}\left(x_{1}, x_{2}, a_{1} x_{1}+b_{1} x_{2}\right)+v B_{2}\left(x_{1}, x_{2}, a_{i} x_{1}+b_{i} x_{2}\right)
\end{array}\right.
$$

We define the homogeneous function of degree 2 in two dimension

$$
\begin{aligned}
& \mathcal{G}_{i}\left(x_{1}, x_{2}\right)=\mathcal{G}\left(x_{1}, x_{2}, x_{3}=a_{i} x_{1}+b_{i} x_{2}\right) \\
& \mathcal{F}_{i}\left(x_{1}, x_{2}\right)=\mathcal{F}\left(x_{1} \cdot x_{2}, x_{3}=a_{1} x_{1}+b_{i} x_{2}\right) \\
& \mathcal{H}_{i}\left(x_{1}, x_{2}\right)=\mathcal{H}\left(x_{1}, x_{2}, x_{3}=a_{i} x_{1}+b_{i} x_{2}\right) .
\end{aligned}
$$

The authors prove the following theorem [7].

\section{Theorem 3}

If there exists a function $\Phi$ satisfying to the following conditions [7]

1. The function $\Phi$ is $C^{\infty}$ on $\mathbb{R}^{2} \backslash\{(0,0)\}$, and homogeneous of degree 2 .

2. The functions $\left(c_{1} x_{1}-\tilde{c}_{1} x_{2}\right)\left(c_{2} x_{1}-\tilde{c}_{2} x_{2}\right)$ divides $\mathcal{H}_{i}(x)+v(x) \mathcal{G}_{i}(x)$

3. If the point $\xi=\left(\xi_{1}, \xi_{2}\right)$ satisfies $\Phi\left(\xi_{1}, \xi_{2}\right)=0$, then $\frac{\mathcal{G}_{i}\left(\xi_{1}, \xi_{2}\right)}{\mathcal{F}_{i}\left(\xi_{1}, \xi_{2}\right)}>0$.

Then the feedback

$$
v_{i}\left(x_{1}, x_{2}\right)=\frac{\phi_{i}\left(x_{1}, x_{2}\right)-\mathcal{H}_{i}\left(x_{1}, x_{2}\right)}{\mathcal{G}_{i}\left(x_{1}, x_{2}\right)}
$$

is $C^{\infty}$ on $\mathbb{R}^{3} \backslash\{(0,0,0)\}$ homogeneous of degree 0 and stabilizes the system in eqn. (8).

\section{Theorem 4}

The system in eqn. (6) is globally asymptotically stable at the origin 
by a homogeneous function of degree 0 if and only if the restriction of $A+v B$ on each invariant plane is globally asymptotically stable at the origin.

Proof: We suppose that the system in eqn. (6) is GAS at the origin by the homogeneous function $u\left(x_{1}, x_{2}, x_{3}\right)$ of degree 0 . According to the hypothesis that the plane $\mathcal{P}_{i}:=x_{3}=a_{i} x_{1}+b_{i} x_{2}$ is invariant by the vector fields $A$ and $B$, the the restriction of $A+u B$ on this plane is also GAS by the feedback $u\left(x_{1}, x_{2}, a x_{1}+b x_{2}\right)$. Inversely, suppose that the restriction of $A+u B$ on each invariant plane is globally asymptotically stable at the origin. In the plane $\mathcal{P}:=x_{3}=a x_{1}+b x_{2}$, the system in eqn.(6) is reduced to the system in eqn. (8). To construct the homogeneous feedback $u_{i}$ of the system (8), we construct a function $\Phi$ satisfying the conditions of theorem, such that the feedback

$$
u_{i}\left(x_{1}, x_{2}\right)=\frac{\phi_{i}\left(x_{1}, x_{2}\right)-\mathcal{H}_{i}\left(x_{1}, x_{2}\right)}{\mathcal{G}_{i}\left(x_{1}, x_{2}\right)}
$$

is $C^{\infty}$ on $\mathbb{R}^{2} \backslash\{(0,0,0)\}$ homogeneous of degree 0 and stabilizes the system in eqn.(8) in the plane $\mathcal{P}_{i}:=x_{3}=a_{i} x_{1}+b_{i} x_{2}$.

The theorem of Coleman say that the origin is asymptotically stable if and only if $\mathrm{v}$ stabilize system in eqn. (6) in all invariant straight line for any periodic solution. So, if $\langle v\rangle$ is an invariant straight line by the flow of the system in eqn. (8), then $A v+u(v) B v=v v$ and

$$
\mathcal{D}\left(v_{1}, \mathrm{v}_{2}, v_{3}\right)=\operatorname{det}\left(\begin{array}{lll}
A_{1}(v) & B_{1}(v) & v_{1} \\
A_{2}(v) & B_{2}(v) & v_{2} \\
A_{3}(v) & B_{3}(v) & v_{3}
\end{array}\right)=0
$$

If

$$
\mathcal{D}\left(x_{1}, x_{2}, x_{3}\right)=\left(x_{3}-a_{1} x_{1}-b_{1} x_{2}\right) R\left(x_{1}, x_{2}, x_{3}\right)
$$

the feedback $u$ take this form:

$$
\left\{\begin{array}{l}
u\left(x_{1}, x_{2}, x_{3}\right)=\frac{\phi_{i}\left(x_{1}, x_{2}\right)-\mathcal{H}_{i}\left(x_{1}, x_{2}\right)}{\left(x_{3}-a_{1} x_{1}-b_{1} x_{2}\right)^{2}+\mathcal{G}_{i}\left(x_{1}, x_{2}\right)} \text { if }\left(x_{1}, x_{2}, x_{3}\right) \neq(0,0,0) \\
u(0,0,0)=0
\end{array}\right.
$$

\section{If}

$\mathcal{D}\left(x_{1}, x_{2}, x_{3}\right)=\left(x_{3}-a_{1} x_{1}-b_{1} x_{2}\right)\left(x_{3}-a_{2} x_{1}-b_{2} x_{2}\right)\left(x_{3}-a_{3} x_{1}-b_{3} x_{2}\right)=p_{1}(x) p_{2}(x)$ $p_{3}(x)$ with $p_{1}(x)=x_{3}-a_{1} x_{1}-b_{1} x_{2}, p_{2}(x)=x_{3}-a_{2} x_{1}-b_{2} x_{2}$ and $p_{3}(x)=x_{3}-a_{3} x_{1}-$ $b_{3} x_{2}$.

The feedback $u$ take this form:

$$
\left\{\begin{array}{l}
u\left(x_{1}, x_{2}, x_{3}\right)=\frac{p_{2}(x) p_{3}(x)\left(\phi_{i}\left(x_{1}, x_{2}\right)-\mathcal{H}_{i}\left(x_{1}, x_{2}\right)\right)}{p_{1}(x)^{2}+p_{2}(x) p_{3}(x) \mathcal{G}_{i}\left(x_{1}, x_{2}\right)}+\frac{p_{2}(x) p_{3}(x)\left(\phi_{i}\left(x_{1}, x_{2}\right)-\mathcal{H}_{i}\left(x_{1}, x_{2}\right)\right)}{p_{2}(x)^{2}+p_{1}(x) p_{3}(x) \mathcal{G}_{i}\left(x_{1}, x_{2}\right)}+ \\
\frac{p_{2}(x) p_{3}(x)\left(\phi_{i}\left(x_{1}, x_{2}\right)-\mathcal{H}_{i}\left(x_{1}, x_{2}\right)\right)}{p_{3}(x)^{2}+p_{1}(x) p_{2}(x) \mathcal{G}_{i}\left(x_{1}, x_{2}\right)} \\
\text { if }\left(x_{1}, x_{2}, x_{3}\right) \neq(0,0,0) \\
u(0,0,0)=0
\end{array}\right.
$$

Under the hypothesis that $\mathcal{P}_{i}:=x_{3}=a_{1} x_{1}+b_{i} x_{2}$ for $i=1,2,3$ is an invariant linear plane of the vector fields $A$ and $B$ then $v_{3}=a_{i} v_{1}+b v_{2}$ and $\varphi\left(v_{1}, v_{2}\right)=0$ Since the zeros of the function $\varphi$ have been constructed to satisfy the second condition of theorem, then the restriction of the closed loop system on the invariant straight line $\langle v\rangle$ is GAS, so, the first condition of The theorem of Coleman is satisfied. For the second conduction of the theorem of Coleman it's clear from theorem that the system in eqn.(6) don't have any periodic orbits. Then, The system in eqn.(6) is globally asymptotically stable at the origin by a homogeneous function of degree 0 .

\section{Example}

We consider the bilinear system

$$
\dot{x}=A x+u B x
$$

where $x=\left(x_{1}, x_{2}, x_{3}\right) \in \mathbb{R}_{3}, u \in \mathbb{R}$ and

$$
A=\left(\begin{array}{ccc}
1 & -2 & 1 \\
-2 & 1 & -2 \\
-2 & 1 & -2
\end{array}\right) \text { and } B=\left(\begin{array}{llc}
2 & 1 & 1 \\
-1 & 2 & -2 \\
-1 & 2 & -2
\end{array}\right)
$$

The considered class satisfy to condition [9]

$$
\operatorname{det}(A x, B x, x)=\left(x_{1}-x_{2}\right)\left(3 x_{1}^{2}+4 x_{1} x_{2}-5 x_{2}^{2}+2 x_{1} x_{3}+4 x_{2} x_{3}+x_{3}^{2}\right)
$$

and the sub manifold $\mathcal{M}$ is defined by

$$
M=\left\{x \in \mathbb{R} \text { such that } x_{3}=x_{2}\right\}
$$

it is easy to see that for $x \in \mathcal{M}$, on has

$$
\begin{aligned}
& \mathcal{G}\left(x_{1}, x_{2}\right)=x_{1}^{2}+2 x_{1} x_{2}+2 x_{2}^{2} \\
& \mathcal{H}\left(x_{1}, x_{2}\right)=x_{2}\left(3 x_{1}-2 x_{2}\right) \\
& \mathcal{F}\left(x_{1}, x_{2}\right)=3 x_{1}\left(x_{1}+2 x_{2}\right)
\end{aligned}
$$

The condition in eqn.(3) of theorem is equivalent to the existence of such $m$ satisfies $\Phi(\mathrm{m})=0$ and $\frac{\mathcal{F}(m)}{\mathcal{G}(m)}>0$

We can chose $m=(1,1)$ and $\Phi\left(x_{1}, x_{2}\right)=\left(x_{1}-x_{2}\right)^{2}$ then we can write

$$
u(x)=\frac{x_{1}^{2}-5 x_{1} x_{2}+3 x_{2}^{2}}{\left(x_{1}-x_{2}\right)^{2}+x_{1}^{2}+2 x_{1} x_{2}+2 x_{2}^{2}}
$$

is homogeneous of degree 0 . Since $A\left(\begin{array}{l}1 \\ 1 \\ 1\end{array}\right)+u(1,1,1) B\left(\begin{array}{l}1 \\ 1 \\ 1\end{array}\right)=-\frac{9}{5}\left(\begin{array}{l}1 \\ 1 \\ 1\end{array}\right)$ then the feedback $u(x)$ stabilizes the system in eqn.(12) in the unique invariant straight line.

\section{References}

1. Bacciotti A, Boieri $P$ (1991) A characterization of single-input planar bilinear systems which admit a smooth stabilizer. Syst Control Lett 16: 139-144.

2. Cesari L, LaSalle J, Lefschetz S (1960) Contributions to the Theory of Nonlinear Oscillations. Princeton university press, 5.

3. Ryan EP (1983) Global asymptotic stabilization of a class of bilinear control system. Int J Control 38: 359-367

4. Jerbi H, Kharrat T, Fawzi O (2016) Global Stabilization of a Class of Bilinear Systems in $\mathrm{R}^{3}$, Mediterr. J Math 13: 2507-2524

5. Jerbi $\mathrm{H}$, Kharrat $\mathrm{T}$ (2003) Asymptotic stabilizability of homogeneous polynomial systems of odd degree. Syst Control Lett 48: 87-99.

6. Jerbi H, Ouled Maaloum A (2007) Feeddack stabilization of homogeneous polynomial systems of odd degree in the plain. Syst Control Lett 56: 611-617.

7. Jerbi H, Kharrat T, Sioud K (2016) Stabilization of Homogeneous Polynomia Systems in the Plane, Kybernetika 52: 131-152.

8. Kawski M (1995) Geometric homogeneity and stabilisation. Nonlinear Control System 1: 164-169.

9. Luesing R, Nijmeijer $H$ (1989) On the stabilisation of bilinear systems via constant feedback. Linear Algebra Appl 122: 457-474 